\title{
Nonlinear features of equatorial baroclinic Rossby waves detected in Topex altimeter observations
}

\author{
R. E. Glazman, A. Fabrikant(*) and A. M. Greysukh \\ Jet Propulsion Laboratory, Califomia Institute of Technology, Pasadena CA 91109, USA \\ (*)Department of Meteorology, Pennsylvania State University, University Park, PA 16802-5013, USA
}

Received 9 October 1995 - Accepted 5 January 1996 - Communicated by A. R. Osborne

\begin{abstract}
Using a recently proposed technique for statistical analysis of non-gridded satellite altimeter data, regime of long equatorially-trapped baroclinic Rossby waves is studied. One-dimensional spatial and spatiotemporal autocorrelation functions of sea surface height (SSH) variations yield a broad spectrum of baroclinic Rossby waves and permit determination of their propagation speed. The 1-d wavenumber spectrum of zonal variations is given by a power-law $k^{-2}$ on scales from about $10^{3} \mathrm{~km}$ to $10^{4} \mathrm{~km}$. We demonstrate that the observed wave regime exhibits features of soliton turbulence developing in the long baroclinic Rossby waves. However, being limited to second statistical moments, the present analysis does not allow us to rule out a possibility of weak wave turbulence.
\end{abstract}

\section{Introduction}

Baroclinic Rossby waves represent an important component of equatorial wave dynamics. Of particular interest is the question about these waves' nonlinearity. One of the goals of the present work is to detect manifestations of the nonlinear nature of equatorially trapped baroclinic Rossby waves.

Weakly-nonlinear long Rossby waves obey the Korteweg-de Vries (KdV) equation and, in a stationary regime, form solitons (Boyd, 1980; Marshall and Boyd, 1987) or "soliton trains" (Boyd, 1991). When their amplitude is sufficiently large, the solitons acquire properties of westward propagating mid-latitude modons (Boyd, 1985). In particular, they carry a region of closed recirculation - an important feature with respect to mass and heat transport in equatorial regions. A set of solitons with randomly distributed amplitudes, hence speeds, is referred to as a soliton gas or soliton turbulence (Kingsep et al., 1973; Gorshkov et al., 1977; D'yachenko et al., 1989). Osborne $(1993,1995)$ showed that a 1-dimensional wave field composed of random solitons (plus a transient component referred to as "radiation"), satisfying the periodic $\mathrm{KdV}$ equation, is characterized by the wavenumber (power) spectrum of form $k^{-\gamma}$. A special case of $\gamma=2$, studied by Osborne (1993) in detail, finds confirmation in our experimental data, Sects. 3 and 5. A brief summary of basic notions related to soliton turbulence is provided in Appendix $B$ to facilitate the interpretation of our experimental findings.

An alternative regime of nonlinear wave dynamics is represented by weak wave turbulence (Zakharov et al., 1992). Spectral cascades of energy, enstrophy and other conserved quantities which are manifested in a cascade pattern in the surface topography are an important feature of this particular regime (Monin and Piterbarg, 1987; Mikhailovskii et al., 1988; Novakovskii et al., 1988; Balk and Nazarenko, 1990). In either case, the characteristic propagation speed of nonlinear waves is greater than that predicted by linear wave theory, and the wavenumber spectrum of sea surface height (SSH) zonal variations tends to be rather broad. One formal distinction between soliton and weak wave turbulence is the degree of phase coupling between individual Fourier components of the wave field: while being weak for wave turbulence, this coupling is strong for solitons; see Appendix B for further discussion. Unfortunately, the present analysis does not allow us to estimate this coupling, although the wavenumber spectrum of zonal SSH variations and other properties of the wave field reported in Sect. 5 suggest that the waves are essentially non-linear.

Using a recently developed statistical approach (Glazman et al., 1996), we examine SSH variations in a narrow zonal channel located at $0.75^{\circ} \mathrm{S}$ in the Pacific. Sampling limitations related to the satellite orbit configuration determine the temporal and spatial resolution of our analysis. As explained in Sects. 2 and 3, we can presently resolve variations with time scales greater than about 10 days and spatial scales starting at about $800 \mathrm{~km}$. Wave motions with smaller scales are filtered out due to the averaging procedure described in Sect. 3. In order to emphasize this filtering property, a remote analogy between our statistical approach and the quasi-geostrophic approximation may be pointed out. While insufficient for studies of baroclinic Kelvin and all types of barotropic 
waves, our technique is adequate for analysis of long baroclinic Rossby waves. Indeed, as shown in Fig. 1, the period of the first meridional, first baroclinic Rossby mode is at least three times as large as the corresponding Kelvin wave period for the same wavelength - i.e., is always greater than 35 days.

As an alternative to a statistical analysis, large-scale zonal motion in equatorial regions can be deduced from a sequence of SSH contour plots by tracking propagation of individual, conspicuous features of the SSH field (e.g., Delcroix et al. 1991; Musman, 1992). However, such a "deterministic" approach permits positive identification of only rather intense events of equatorial waves, on a case-bycase basis. Altimeter measurement errors, not suppressed by averaging over a large data sample, make it difficult to resolve small-amplitude SSH oscillations (under $5 \mathrm{~cm}$ or so). Moreover, the plots of an "instantaneous" SSH field, based on observations over a large time interval (at least 17 days in the case of Geosat altimeter measurements and at least 10 days for Topex measurements), do not permit an accurate determination of the shape of observed waves.

The satellite data and their reduction are described in Sect. 2. Our statistical technique is sketched in Sect. 3. Section 4 reviews properties of autocorrelation functions important for our analysis. Results of the data analysis are reported in Sect. 5. In Appendix A we present linear model predictions for the autocorrelation function of a broad-band system of baroclinic Rossby and Yanai waves. This facilitates the interpretation of the statistical quantities in Sect. 6. Conclusions are summarized in Sect. 7.

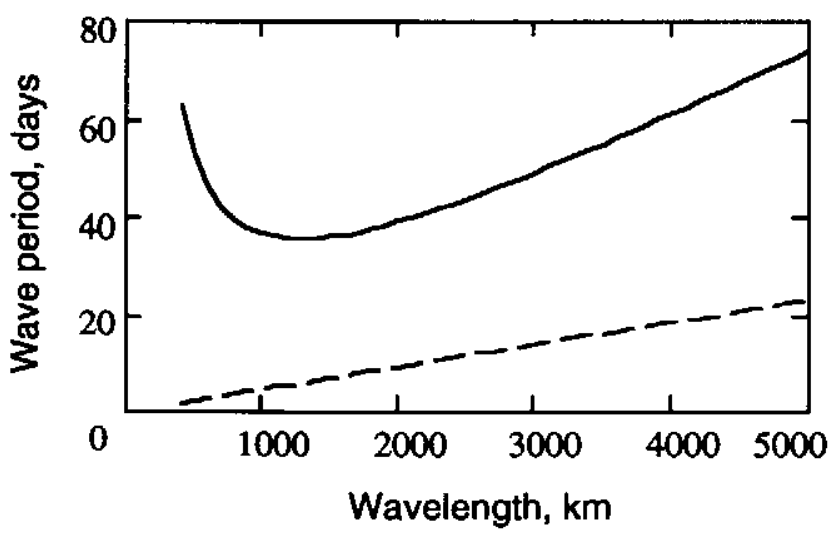

Fig. 1. Period of linear baroclinic Rossby (solid curve) and - Kelvin (dashed curve) waves (1 st baroclinic, 1st meridional mode) versus wavelength, based on Eq.(A1). The Kelvin wave speed is assumed to be $2.5 \mathrm{~m} / \mathrm{s}$ and the (1 st baroclinic) Rossby radius of deformation $250 \mathrm{~km}$.

\section{Dataset of SSH observations and data subsetting}

As described in (Benada, 1993), Topex altimeter data contain a number of standard corrections. For our application, the most important ones are the static atmospheric pressure ("inverse barometer"), electromagnetic ("sea state") bias, tidal (solid Earth and ocean) and geoid. The residual r.m.s. error in SSH measurements is presently believed to be about $5 \mathrm{~cm}$ (Fu et al., 1994). In

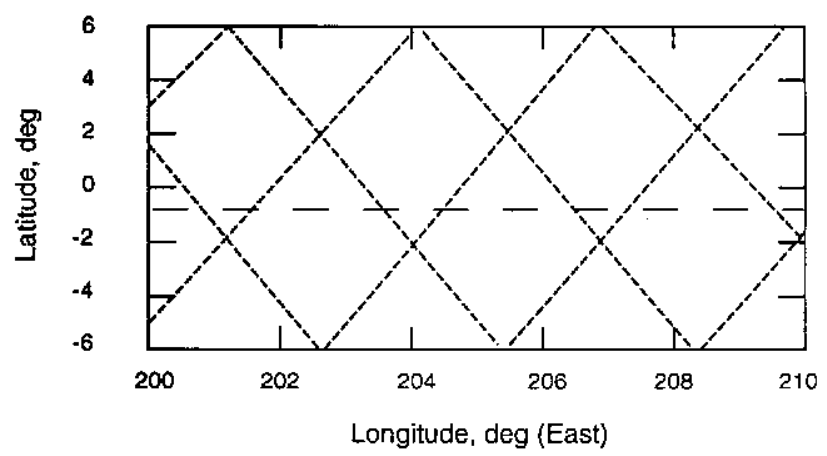

Fig. 2. Satellite ground tracks in a tropical Pacific region during a 10-day cycle. A horizontal dotted line illustrates the position of a zonal string: the SSH data are sampled from groundtracks at intersections with the string.

addition to the standard corrections, we introduce an orbit correction in the fashion of (Tai, 1989), as discussed in detail in (Glazman et al., 1996). Finally, we remove the time-invariant trend in the SSH field. This is done by subtracting the mean (over the period of observations) SSH value at each spatial point from a current value. Usually this procedure is employed to eliminate remaining uncertainties in the Earth geoid. In our case, it also serves to remove spatial trends due to stationary features of ocean dynamics. Specifically, it eliminates a stationary component associated with the mean east-west slope of the sea level (caused by Trade winds) along the equator. Our check of the thus processed data showed the remaining linear trend along the equator to be $3 \times 10^{-5} \mathrm{~cm} / \mathrm{km}$, i.e. practically absent. The dataset includes 530 days worth of Topex observations, covering cycles 13 through 65 - the June 93 -Aug. 94 period characterized by a pronounced sequence of Rossby waves.

We consider SSH spatial and temporal variations in a $12,200 \mathrm{~km}$ long zonal channel centered on the equator. SSH measurements are spaced roughly $6 \mathrm{~km}$ along the altimeter groundtracks. Figure 2 illustrates geometry of the tracks for a small area of the equatorial Pacific. Apparently, the minimal spatial separation of SSH measurements in the zonal direction depends on the latitude. At $\pm 2^{0}$ off the equator this separation is down to about 10 $\mathrm{km}$. However, on the equator this distance exceeds $150 \mathrm{~km}$. This factor, along with some additional requirements described in the next section, constrains the spatial resolution of our analysis.

Altimeter SSH measurements from all ascending and descending passes, extracted at fixed latitudes, were grouped into 1-dimensional strings of data points aligned along the equator. These zonal strings are spaced about $5 \mathrm{~km}$ in the meridional, i.e., y - direction. To estimate spatio-temporal autocorrelation function $W(r, \tau)$ at a given latitude, we employed four adjacent zonal strings centered about this latitude. The spatial lag, r, is along the equator. The autocorrelation functions calculated for individual strings in a group were ultimately averaged - to increase the statistical significance of the $W(r, \tau)$ estimation. The mean $W(r, \tau)$ was referenced to the central latitude of the group. Therefore, our spatio-temporal autocorrelation function represents an average for a zonal "sub-channel" $20 \mathrm{~km}$ wide. The analysis presented in the following sections employs 
four strings centered at about $0.75^{0} \mathrm{~S}$. The choice of this particular latitude is based on the fact that the amplitude ratio of the first meridional mode to the second mode attains a local maximum here. The shape of the autocorrelation function estimated for other strings was essentially the same.

\section{Evaluation of spatio-temporal autocorrelation functions and power spectra}

Accurate wavenumber-frequency spectra $\Phi(\omega, k)$ would be most useful for analysis of wave processes. However, practical estimation of $\Phi(\omega, \mathrm{k})$ requires data on a regular space-time grid. The actual format of satellite altimeter data is not suited for the task, and interpolating the SSH data onto a regular grid would drastically degrade the spatial and temporal resolution of spectral analysis. Autocorrelation functions $W(r, \tau)$ provide an effective alternative, for they are much easier to estimate based on non-gridded data. Their use for analysis of wave processes is explained in the next section.

For each zonal string, the data were analyzed in the following manner. In order to estimate spatio-temporal autocorrelation function $W(r, \tau)$, we calculated SSH products $\eta(x, t) \eta(x+r, t+\tau)$ for all possible pairs of points on the $(x, t)$ plane and grouped them by values of spatial and temporal lags into $(\Delta r, \Delta \tau)$ bins on the $(r, \tau)$ plane. Ultimately, all products within each bin are averaged. Therefore, the absolute times and longitudinal positions of individual measurements are "forgotten." Strictly speaking, this is justified only for a statistically stationary and spatially homogeneous random function $\eta(x, t)$. The assumption of spatial statistical homogeneity and stationarity implicit in our analysis is quite common in ocean and atmosphere studies. However, possible effects of non-stationarity remain largely unknown, hence the results should be treated with some caution.

The minimum size $\Delta r$ of the spatial-lag bins is constrained by the longitudinal separation of satellite groundtracks. We selected $\Delta \mathrm{r}=350 \mathrm{~km}$ as the optimal size. The choice of $\Delta \tau$ is based on the following compromise. On the one hand, the greater this interval, the more SSH products become available for estimating the autocorrelation function on the r-grid for a given $\tau$. On the other hand, if interval $\Delta \tau$ is large, the distance traveled by an ocean wave during time $\Delta \tau$ may well exceed the size of the $\Delta r$-bin. This would distort the autocorrelation function. Obviously, for studies of Rossby waves, we should choose $\Delta \tau \leq \Delta r / C_{R}$ where $C_{R}$ is the characteristic phase speed of baroclinic Rossby waves $\left(C_{R} \approx 1 \mathrm{~m} / \mathrm{s}\right)$. The surface's variations during this time interval can be neglected. Therefore, $\Delta \tau$ is called the "synchronicity interval." The averaging over $\Delta \tau$ bins filters out SSH oscillations with periods smaller than $2 \Delta \tau$. Most of the results described in Sects. 5 and 6 are obtained with $\Delta \tau=4$ days. Obviously, the choice of $\Delta r=350 \mathrm{~km}$ and $\Delta \tau=4$ days is quite adequate for analysis of baroclinic Rossby waves. However, information on gravity and Kelvin waves will be suppressed because their wave period is relatively small. In reality, Kelvin waves would travel $860 \mathrm{~km}$ in 4 days, hence they would cross two $\Delta r$-bins. Their effect on the autocorrelation function $W(r, 0)$ is illustrated later in this section.

The averaging of all SSH products within the $(\Delta r, \Delta \tau)$ cells yields an unbiased estimate of the autocorrelation function $W(r, \tau)$ on the regular $r-\tau$ grid. Error analysis for this technique is presented in (Glazman et al., 1996). The averaging suppresses the impact of altimeter measuring errors and has other advantages. The r.m.s. error of the autocorrelation function estimate, caused by $\mathrm{SSH}$ measuring errors, is $\Delta_{\mathrm{eW}} \approx \mathrm{e}^{2} / \sqrt{\mathrm{N}}$ where $\mathrm{N}$ is the mean number of independent products $\eta(x) \eta(x+r)$ in $\Delta r$ bins and $e$ is the r.m.s. error of SSH measurements. For $\mathrm{e} \approx 5 \mathrm{~cm}$ and $N=3 \times 10^{3}$, we find $\Delta_{\mathrm{e}} \mathrm{W} \approx 0.5 \mathrm{~cm}^{2}$. The histogram of the number, $N(r)$, of SSH products falling into $\Delta r$ bins is presented in Fig. 3 for $\tau=0$, for several values of $\Delta \tau$. The fact that this distribution is highly non-uniform complicates estimation of error bars. However, it is easy to see that, with the lowest values of $N(r)$ being of order $10^{3}$, the overall accuracy of our statistical technique is sufficient to study SSH variations with the amplitude as small as 1 $\mathrm{cm}$. A more detailed description of our statistical approach is provided in (Glazman et al., 1996).

Figure 4 illustrates an effect of the synchronicity interval on the spatial autocorrelation function $W(r)$, for a full-length zonal sub-channel centered at $y=0.75^{\circ} \mathrm{S}$. The diamond represents the total variance of SSH, $\left\langle\eta^{2}\right\rangle=$ W(0), for which both temporal and spatial lags are exactly zero. The points connected by the solid curve have the synchronicity interval ranging from 2 to 10 days (panels A through D); the actual time difference in individual SSH products is always greater than zero: point $\left\langle\eta^{2}\right\rangle$ is not included into the curve (and is also ignored in the following discussion) because this point is dominated by a deltacorrelated (i.e., "white") noise due to instrumental and measuring errors.

On a close examination of panels $C$ and $D$ in Fig. 4, one can notice a slight eastward displacement (about 600 $\mathrm{km}$ in Panel C) of the peak of W(r). We explain this shift by the influence of baroclinic Kelvin waves. With a 7-day synchronicity interval, Kelvin waves will make their largest positive contribution to $W(r)$ at spatial lags $r \approx c_{K} t$ where $0<t<7$ days and $c_{K}$ is the Kelvin phase speed. Consider a Kelvin-wave-induced component, $W_{K}(r)$, of the estimated autocorrelation function $W(r)$. Effect of a synchronicity interval, $T$, is found by averaging the true spatio-temporal autocorrelation function, $K(r, \tau)$, of Kelvin waves over all time-lags within $T$. For simplicity we assume the distribution of the time lags to be uniform, which yields:

$$
W_{K}(r)=\frac{1}{T} \int_{0}^{T} K\left(r-c_{K} \tau\right) d \tau=\frac{1}{c_{K} T} \int_{-c_{X} T / 2}^{c_{K} T / 2} K(\rho-\xi) d \xi
$$

Here we introduced $\xi=c_{K} \tau-c_{K} T / 2$ and $\rho=r-c_{K} T / 2$. As a function of $\rho$, this autocorrelation function is even: $W_{K}(\rho)=W_{K}(-\rho)$, which yields $r_{m}=c_{K} T / 2$ for the point at which $W_{K}(r)$ attains its maximum. With $T=7$ days and $c_{K}=2.5 \mathrm{~m} / \mathrm{s}$, we find $r_{m} \approx 750 \mathrm{~km}$. The importance (if not the dominance) of the Kelvin-wave-induced component of $W$ at these small time lags explains why this $r_{m}$ is in a reasonable agreement with Fig. 4(C).

The spatial autocorrelation function, Fig. 4, shows a near-linear behavior for large spatial scales -- from about 

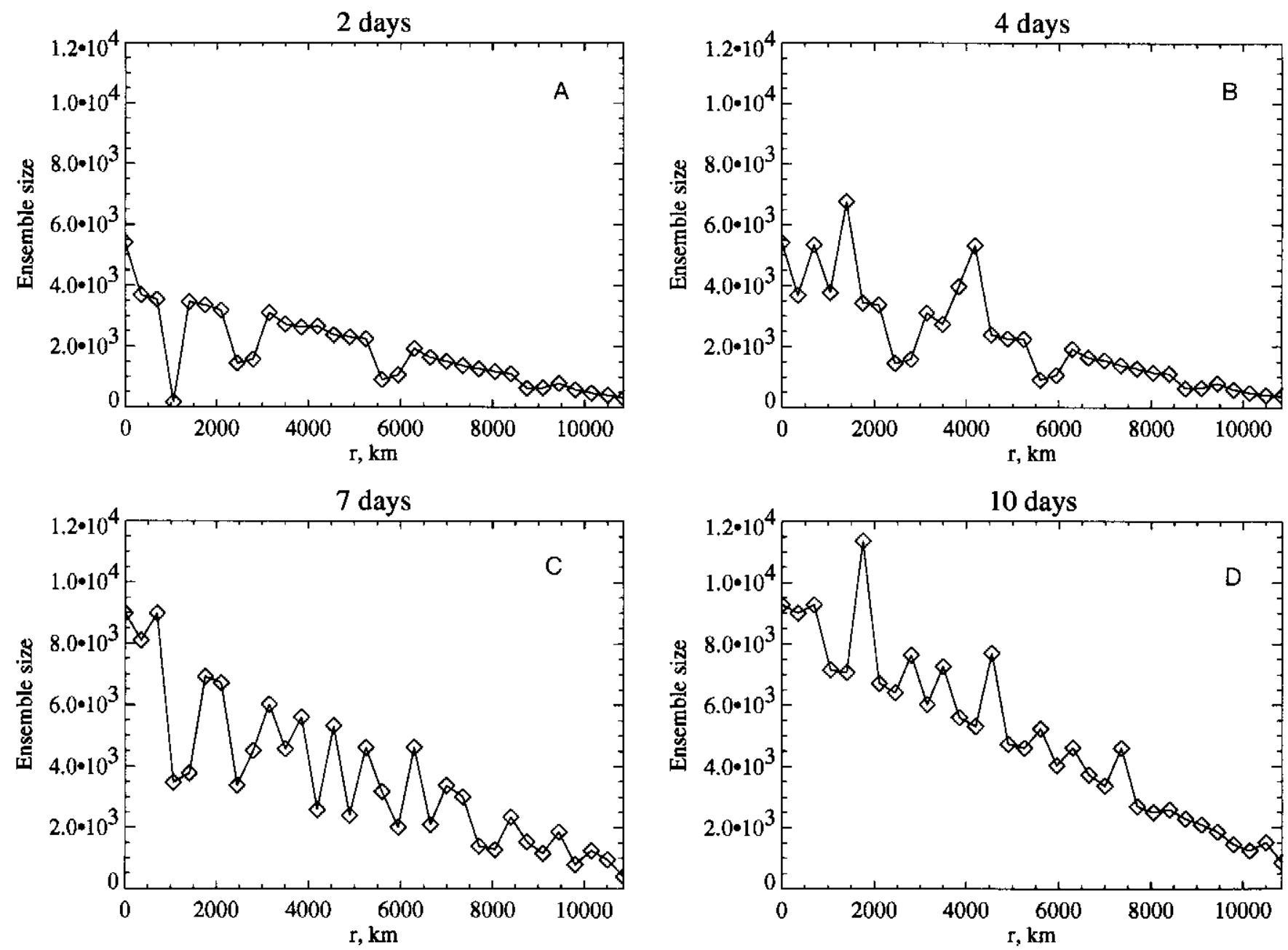

Fig. 3. Number $N(r)$ of $S S H$ products falling into $350 \mathrm{~km}$ r-bins for $W(r, 0)$ of the full-length zonal string. Values of the synchronicity interval are provided on top of each panel.

1000 to $9000 \mathrm{~km}$. The corresponding shape of the wavenumber spectrum is inferred based on the following argument. The 1- $d$ wavenumber spectrum of form

$$
F(k) \propto k^{-3+2 \mu},
$$

with $\mu>0$, yields the following asymptotic form of the spatial autocorrelation function:

$$
W(r)=W(0)-B r^{2-2 \mu},
$$

[e.g., (Glazman and Weichman, 1989)]. Here, B is a constant. Hence, when $W(r)$ is a linear function $(\mu=1 / 2)$, the spectrum follows power law $F(k) \sim k^{-2}$ in the corresponding range of wavenumbers.

The spatio-temporal autocorrelation function for the Pacific equator (at $0.75^{\circ} \mathrm{S}$ ) is illustrated in Fig. 5 .

In principle, autocorrelation functions permit evaluation of power spectra. However, random errors in the estimated values of $W(r, \tau)$ and the rather narrow range of $r$ and $\tau$ for which $W(r, \tau)$ is available from altimeter data greatly limits the accuracy of the corresponding Fourier transform. Therefore, the direct use of autocorrelation functions for analyzes of wave properties has great advantages.

\section{Using autocorrelation functions for analysis of wave processes}

The spatio-temporal autocorrelation function, Fig. 5, contains a great deal of information on processes occurring within our 530-day period of observations. For example, let us notice that the secondary ridge of $W(r, \tau)$ is separated from the main one by $\tau=1$ year. This ridge (and its mirror image at negative $\tau$ ) is associated with the annual variability rather than the intrinsic period of Rossby waves. Most relevant to our problem is the main ridge of $W(r, \tau)$, especially the part for which the lag is well within 100 days -- the time required for long baroclinic Rossby waves to freely traverse the equatorial channel at the speed $=1 \mathrm{~m} / \mathrm{s}$. For this range of $\tau, W(r, \tau)$ is related to the wavenumber spectrum of SSH zonal variations by

$$
W(r, \tau)=\int F(k) e^{i k[r-c(k) \tau]} d k,
$$

where $\mathbf{c}(\mathbf{k})$ is the wave phase velocity. For a narrow-band spectrum $F(k)$, the integral can be evaluated by the stationary phase method. This would immediately demonstrate that the most prominent feature of the 2 - 

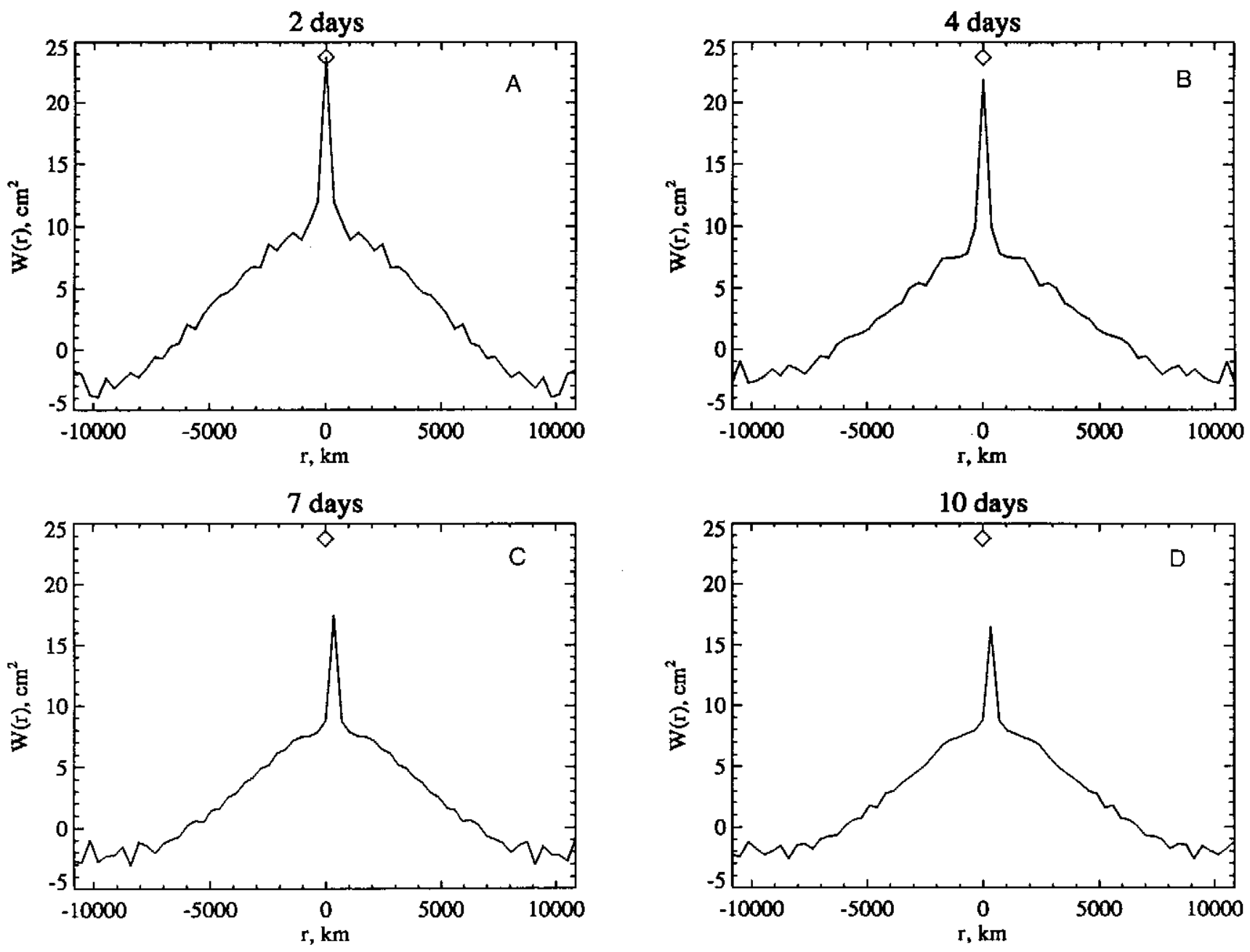

Fig. 4. One-dimensional spatial autocorrelation function, $W(r)$, for the area $160^{\circ}-270^{\circ} \mathrm{W}$ referenced to latitudinal position $y=$ $0.75^{\circ} \mathrm{S}$, estimated for several values of the synchronicity interval $\Delta t$, as indicated on top of each panel. The diamond represents $W(0) \equiv\left\langle\eta^{2}\right\rangle$. The solid curve approximating W(r) ignores this special point because this is the only point for which both $r$ and $\tau$ are exactly zero (see Sects. 3 and 5 for detail).

dimensional function $W(r, \tau)$ is a "ridge" on the $r-\tau$ plane passing through the origin. Its orientation yields the propagation speed of a wave train, d $\omega / \mathrm{dk}$. The latter, of course, is the wave group velocity. (If the spectrum were sufficiently narrow, a system of parallel ridges of a rapidly decreasing height would also be visible on this plane.) In a non-dispersive case $(\mathbf{c}(\mathbf{k})$ is const), (3) reduces to

$$
W(r, \tau)=W(r-c \tau)
$$

In other words, $W(r, \tau)$ at time $\tau$ is obtained from $W(r, 0)$ by a uniform shift along $r$. The wave propagation speed, c, is found as

$$
c=\frac{r(\tau)}{\tau}
$$

Here, $r(\tau)$ is the position of the correlation maximum at time $\tau$. Obviously, Eq.(4) remains also valid in the case of wave solitons because wave dispersion is exactly balanced by effects of wave non-linearity: the wave shape travels without distortions.

For a broad-band spectrum, such as (1) with $\mu>0$, the interpretation of the autocorrelation function is not trivial.
In Appendix A, we carry out numerical simulations of $W(r, \tau)$ for a special case of linear Rossby waves whose spectrum contains a power-law range $\mathrm{k}^{-2}$.

\section{Statistical analysis of baroclinic Rossby waves}

\section{a) Spatial autocorrelation function and wavenumber spectrum}

Figures 4 and 5 point to the existence of two regimes of SSH spatial variations. The small-scale variations responsible for a narrow peak at the origin have spatial scales under $1000 \mathrm{~km}$ and are characterized by $S S H$ variance $\left\langle\eta^{2}\right\rangle \approx 16 \mathrm{~cm}^{2}$ estimated as $W(0)-W(\Lambda)$ where $\Lambda$ is the characteristic spatial lag $\left(\Lambda=10^{3} \mathrm{~km}\right)$ at which $W(\mathrm{r})$ starts displaying the linear trend, and $W(0)$ is presented in Fig. 4 by a diamond. The large-scale variations (corresponding to the linear trend in $W(r)$ ) have spatial scales greater than about $1000 \mathrm{~km}$. Their variance, $\left\langle\eta^{2}>\approx 8 \mathrm{~cm}^{2}\right.$, is found 


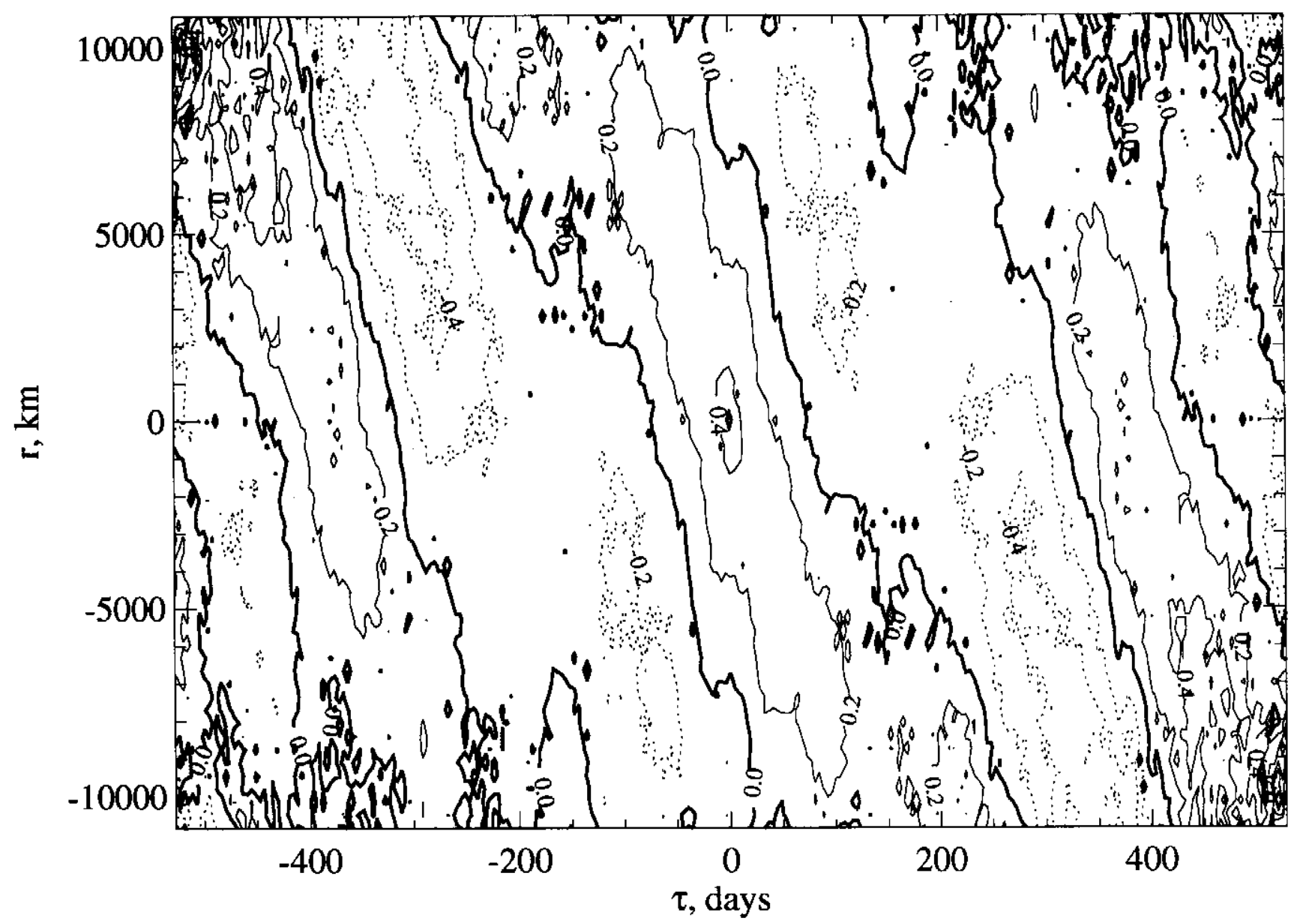

Fig. 5. Spatial-temporal autocorrelation coefficient, $W(r, \tau) / W(0,0)$, for $y=0.75^{0} \mathrm{~S}$. Here, $\mathrm{W}(0,0)=24 \mathrm{~cm}^{2}\left(\equiv\left\langle\eta^{2}>\right)\right.$. Bold contours correspond to $W(r, \tau)=0$.

as $W(\Lambda)$. A more formal evaluation of the SSH variance for large spatial scales can be done by fitting a parabola a $0^{-}$ $a_{2} r^{2}$ to several points with $|r|>1000 \mathrm{~km}$ surrounding the W(r)'s narrow peak: its maximum, a0, provides the desired result.

In accord with (1) and (2), the linear behavior of W(r) on scales greater than $10^{3} \mathrm{~km}$ corresponds to the $\mathrm{k}^{-2}$ range in the 1-d power spectrum $F(k)$. The power spectrum for this large-scale regime can be idealized as

$$
F(k)=\text { const } \cdot e^{-\left(k_{0} / k\right)^{2}} e^{-\left(k / k_{\infty}\right)^{2}} k^{-2}
$$

where the exponential factors serve as the high- and lowpass filters, respectively: $k_{0}$ representing the lowerwavenumber boundary of the linear range and $k_{\infty}$ serving as the "microscale" or the "inner scale" of the spectrum [see, e.g., (Glazman and Weichman, 1989) for a discussion of this type of spectra]. The use of a low-pass filter is necessary only for the study of the SSH realizations (reported in Sect. 6) -- to ensure the convergence of an improper integral. To better understand the narrow peak of $W(r)$ associated with small-scale variations of SSH one has to analyze the temporal evolution of $W(r, \tau)$.

\section{b) Spatio-temporal autocorrelation function}

Figure 6 illustrates several sections of $W(r, \tau)$ for fixed values of the temporal lag. Ignoring for a moment the evolution of $W(r ; \tau)$ near its peak, we can estimate the speed of the westward propagation using (5). Based on the last three panels in Fig. 6 - with $\tau=40,60$ and 80 days - we find $\bar{c} \approx 1.1 \mathrm{~m} / \mathrm{s}-$ in agreement with previously known estimates for baroclinic Rossby waves, see e.g. (Delcroix et al., 1991) and references therein.

A more formal estimation of this speed can be done, for instance, as follows. For a fixed value of $\tau$ onc can estimate the $r$-coordinate of the autocorrelation maximum by approximating $W(r ; \tau)$ by a quadratic polynomial $a_{0}+a_{1} r+a_{2} r^{2}$ and solving equation $\partial W / \partial r=0=a_{I}+2 a_{2} r$ for $r$. A set of such points on the r- $\tau$ planc allows one to fit a straight line $r(\tau)=c \tau$ which yields an estimate of the wave propagation speed, $c$. With step $\Delta \tau=4$ days (for values of $\tau$ from 20 to 80 days), this procedure yields $\mathrm{c}=$ $1.11 \pm 0.22 \mathrm{~m} / \mathrm{s}$ where the uncertainty of $0.22 \mathrm{~m} / \mathrm{s}$ is taken as the standard deviation of experimental points from the straight line $r(\tau)=\bar{c} \tau$. Since the total number of such points is $n=(80-20) / 4=15$, we estimate the confidence intervals as $\Delta \mathrm{c}=\mathrm{t} \sigma / \mathrm{Vn}$ where $\sigma$ is the standard deviation, $\sigma=0.22 \mathrm{~m} / \mathrm{s}$, and $t$ is found from Table 26.10 of 

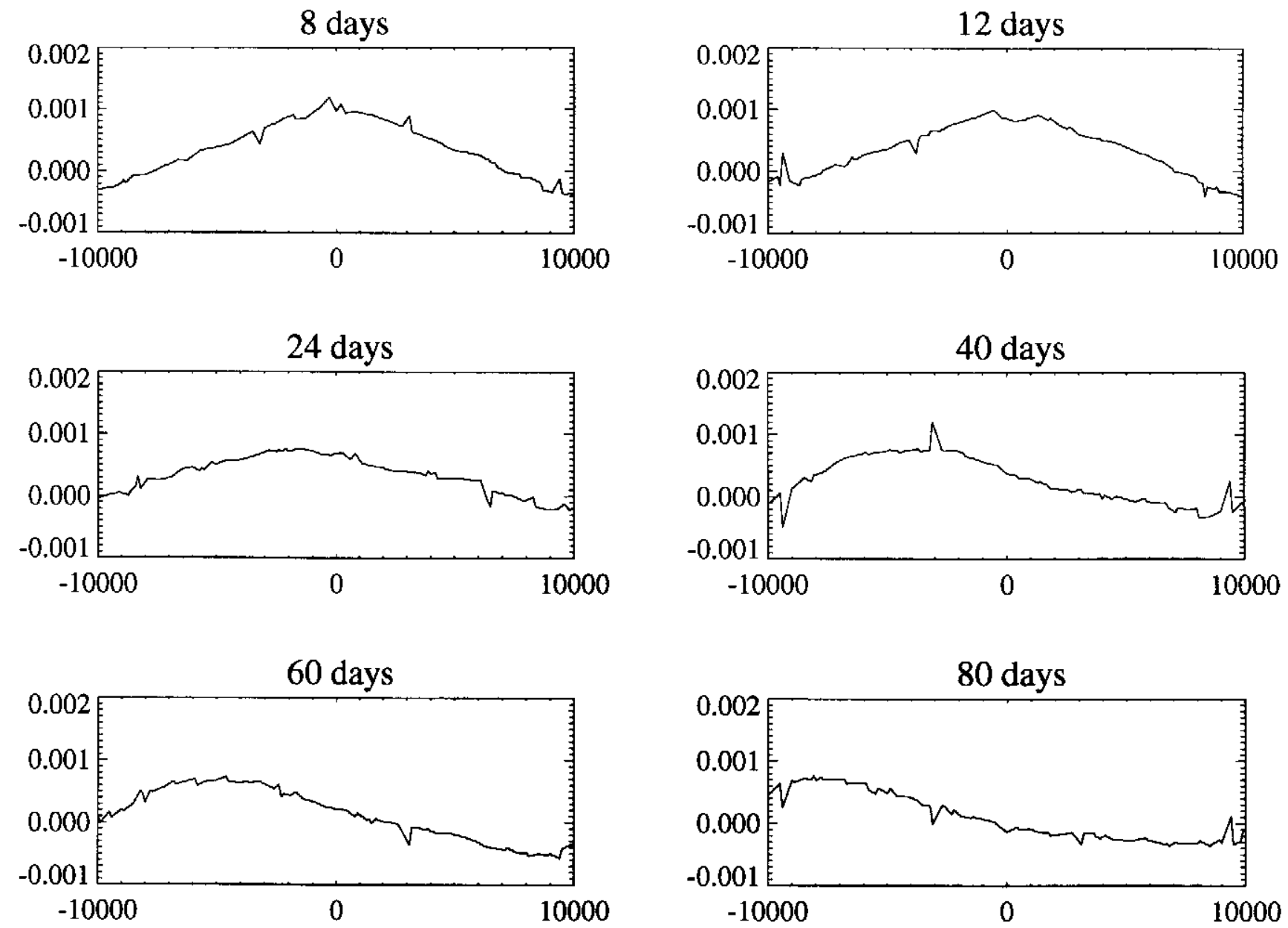

Fig. 6. Spatial-temporal autocorrelation function, $W(r, \tau)$, for $y=0.75^{\circ} \mathrm{S}$, for selected time lags, $\tau$, as designated on top of each panel. Horizontal axis: $r(\mathrm{~km})$. Vertical axis: $W(r, \tau)\left(m^{2}\right)$. Synchronicity interval $\Delta t=4$ days.

(Abramowitz and Stegun, 1970) for a specified value of the significance level, $A$. Taking $A=0.9$, we find $\Delta c=0.1$ $\mathrm{m} / \mathrm{s}$. Therefore, at the 90 percent significance level, we have $\mathrm{c}=1.11 \pm 0.10 \mathrm{~m} / \mathrm{s}$.

If the wavenumber spectrum were narrow, $\bar{c}$ could be viewed as the wave group velocity of baroclinic Rossby waves corresponding to the spectral peak wavenumber. In our case, $\bar{c}$ strongly depends on the spectrum shape. Hence, its usefulness as a characteristic of the equatorial waveguide is not very high. In this respect, the Kelvin wave speed represents a more fundamental quantity, for Kelvin waves are non-dispersive and their velocity is determined by the Brunt-Väisälä frequency. A detailed analysis of this issue is presented in the next section.

As evident from Figs. 4 through 6, the sharp peak in $W(r, \tau)$ is confined to the shortest temporal and spatial scales. These short-scale variations may be caused by the following factors:

1) SSH measuring errors, such as the electromagnetic (sea state) bias caused by wind-generated surface gravity waves. With respect to the coarse spatial and temporal resolution of our technique, this factor represents a deltacorrelated noise, hence it contributes only to $W(0,0)$.
2) High-frequency SSH oscillations due to barotropic and fastest baroclinic gravity wave modes. Considering the relatively large size of the time-lag bins, $\Delta \tau$, contribution of these fast waves is similar to that of a delta-correlated noise: they increase $W(r, \tau)$ only for smallest values of $\tau$.

3) Kelvin waves. Similar to other high-frequency oscillations, Kelvin waves - due to their short wave period ( 2.4 days for a $500 \mathrm{~km}$ wavelength) - would require a very small synchronicity interval for their detection. Although these waves are not observed in Figs. 5-6 (because of the large lag bins used in our analysis), they manifest themselves in the eastward shift of the W(r) peak in Fig. 4 panels $C$ and D -- as explained in Sect. 3.

4) Short Rossby waves. These are defined as the waves whose wavelength is shorter than $2 \pi R$ where $R$ is the Rossby radius of deformation. Considering the relatively large size of the spatial-lag bins, effect of short (although very slow) Rossby waves is analogous to that of fast waves: both manifest themselves as a deltacorrelated noise, although the short Rosshy waves represent a spatial noise. Therefore, they could increase $\mathrm{W}(r, \tau)$ only at short spatial lags $r$. 
For our analysis of long baroclinic Rossby waves, all these small-scale processes present little interest.

\section{Interpretation of the results}

Since we have no information on higher statistical moments of the SSH field, the interpretation of Figs. 4 - 6 depends on our assumptions regarding the phase coupling between Fourier components comprising the power spectrum. The simplest assumption is that the SSH variations represent a Gaussian random field. In particular, there is no phase coupling between the Fourier components - the waves are linear. In Appendix A, a linear-wave analysis is presented to relate the propagation speed, $\bar{c}$, of the autocorrelation maximum, which was estimated based on our SSH data, to the phase speed, $c_{R, 1}(0)$, of longest (non-dispersive) baroclinic Rossby waves. In this section we show, in particular, that the observed $\bar{c}$ is higher than predicted by linear theory. This and other arguments presented in this section lead us to suggest that linear theory of baroclinic Rossby waves is at odds with our observations. An alternative interpretation is offered based on a view of equatorial Rossby waves as a sequence of solitons or equatorial modons.

Most of the earlier experimental studies reported the value of the Kelvin wave speed, $c_{K}$, rather than the Rossby wave speed. Indeed, $c_{K}$ is a convenient intrinsic property of the equatorial waveguide because it is unambiguously related to the Brunt-Väisälä frequency. In order to express our experimental quantity $\bar{c}$ in terms of the Kelvin speed, we need the following theoretical relationship between $c_{K}$ and the phase speed, $c_{R, m}(0)$, of the longest Rossby waves of the $\mathrm{m}$-th meridional mode (e.g., LeBlond and Mysak, 1978):

$$
c_{K}=-(2 m+1) c_{R, m}(0)
$$

where $c_{R, m}(k)$ is the phase speed of the m-th Rossby mode for wavenumber $k$. Equation (A8) shows that $c_{R, m}(0)$ must be about 1.15 times as high as the propagation speed of the autocorrelation maximum. Using $\bar{c}=1.1 \mathrm{~m} / \mathrm{s}$ this yields $c_{R, m}(0)=1.2 \mathrm{~m} / \mathrm{s}$. According to (7), our measured $\bar{c}$ would correspond to the Kelvin speed of $3.6 \mathrm{~m} / \mathrm{s}$. This value is well outside the range of typical Kelvin speeds ( 2.3 to $2.8 \mathrm{~m} / \mathrm{s}$ ) reported for the equatorial Pacific.

The hybrid, Rossby-gravity mode, $m=0$, (also known as the Yanai wave) does not agree with our observations either. As shown in Appendix A, this mode is inconsistent with the shape of $W(r, \tau)$ in Fig. 6 .

Of course, one may try explaining the high valuc of $c_{R, 1}(0)$ by possible inaccuracies of our data analysis. However, we believe that a more relevant explanation can be suggested based on nonlinear theories of equatorially. trapped waves.

Let us show that a soliton gas hypothesis is consistent with the observed autocorrelation function of SSII variations. As evident from Fig. 6, the peak value of
$W(r, \tau)$ established at $\tau \approx 20$ days does not drop as the time lag continues to increase. For dispersive waves this can happen only if the effect of wave dispersion is counteracted by the effect of wave non-linearity, as is the case for Rossby solitons; whereas the linear theory predicts a monotonic decrease of the $W(r, \tau)$ peak value with an increasing $\tau$, Fig. A1. The other argument in support of the soliton gas hypothesis is that the longest waves in the spectrum, being near the basin length size, would be affected by the western boundary which would prevent their propagation as either free or forced progressive waves. However, as the Fourier components of a compact, soliton-like formation, these waves cause no controversy.

Realizations of a non-Gaussian random field with power spectrum (6) can be constructed based on the following idealization. Let the instantaneous wave field $\eta(x ; t)$ bc comprised of surface disturbances ("solitons"), $\eta_{\mathrm{n}}$ :

$$
\eta(x)=\sum_{n} \eta_{n}\left(x-x_{n}\right)
$$

satisfying condition $\eta_{n}(x) \rightarrow 0$ at $|x| \geq L$ where $L$ characterizes the width of a soliton. Let us also assume the solitons to be well separated in space, i.e. $\left|x_{n}-x_{n-1}\right|>>$ L. To make this consideration even simpler, we can assume all individual solitons in (8) to be identical in shape and size. (A more general case is discussed in Appendix B.) Fourier transform $f(k)$ of surface profilc $\eta(x)$ is approximated by the Fourier transform of a single soliton $\eta_{n}$ times a constant which is inversely proportional to the number of solitons per unit length of the surface. Obviously, this Fourier transform is related to the power spectrum (6) by $F(k) \propto[f(k)]^{2}$. Therefore, a segment of the surface profile containing one soliton is found as the

real part of $(2 \pi)^{-1} \int_{-\infty}^{\infty}[F(k)]^{1 / 2} e^{-i k x} d k$. This profilc

is illustrated in Fig. 7. Unfortunately, in the small vicinity of its peak, the shape of this "soliton" is affected by our choice of the low-pass filter in (6). Not knowing the "amplitude" (in terms of the thermocline depih displacement) of these disturbances, we cannot indicate their theoretical propagation speed. However, the fact that this speed exceeds the phase speed of linear Rossby waves indicates that a Rossby soliton (or its higher-nonlinear version - the modon) is consistent with our observations.

The theoretical shape, (B1), of Rossby solitons (Boyd, 1980) is slightly different from that in Fig. 7. Ilowever, since the width, amplitude and spacing of actual solitons vary, the above analysis should not be viewed literally as a derivation of the mean shape. This shape also depends on statistical distributions of the quantities entering (8). Our main goal here was only to show that a broad-band spectrum, such as $\mathbf{k}^{-2}$, may be produced by single-hump formations whose horizontal extent is comparable to that of Rossby solitons. Additional considerations regarding the soliton gas hypothesis are reviewed in Appendix B.

Alternative explanations are also possible. In particular, one could argue that the observed spectrum $\mathrm{k}^{-2}$ is the result of weak turbulence of Rossby waves. Present theorics of Rossby wave turbulence (e.g., Mikhailovskii ct al. 1988; Novakovskii et al., 1988; Balk and Nazarenko, 1990) for 


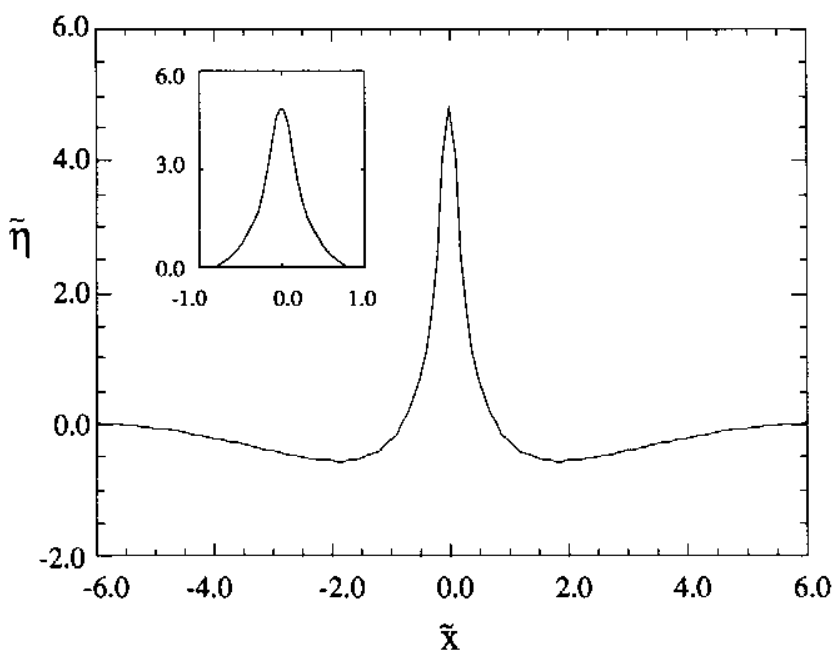

Fig. 7. Non-dimensional surface profile, $\tilde{\eta}(\tilde{x})$, for spectrum (6) with $k_{0} / k_{\infty}=0.1$. Sea surface is assumed to be a sequence, (8), of single-hump disturbances (the "soliton gas"). Vertical axis: arbitrary units of nondimensional length. Horizontal axis is scaled as $\tilde{x}=x k_{0}$ where $\mathrm{x}$ is the dimensional distance along the equator and $k_{0}$ is the lowwavenumber cutoff introduced in (6). Therefore, $\bar{x}=1$ corresponds to $x=1500 \mathrm{~km}$. Inset: the expanded region in the vicinity of $\bar{x}=0$.

long weakly-dispersive waves yield spectra which (in terms of the potential energy distribution relevant in our SSII analysis, and for the zonal wavenumber) fall off as $\mathrm{k}^{-3 / 2}$. Although this prediction is rather close to our observations, some troubling questions regarding their physical realizability and locality of the corresponding Kolmogorovtype cascades arise.

\section{Conclusions}

Equatorially-trapped waves represent a rich dynamical system characterized by many degrees of freedom. Statistical analysis of ocean wave observations offers a natural way of studying such systems. This is especially true with satellite-altimeter data because their temporal and spatial resolution and measuring accuracy are not very high, whereas the geographic and time coverage, for our goals, is practically unlimited. The technique employed in the present work allowed us to detect some new important features of baroclinic Rossby waves.

Our main finding is that, in a wide range of scalcs (from about $10^{3}$ to $10^{4} \mathrm{~km}$ ), zonal spectra of equatorial Rossby waves exhibit a power-law behavior $F(k) \sim k^{-2}$. Our present interpretation of these waves as a sequence of baroclinic Rossby solitons is tentative, for we have no information on higher-order statistics of the wave licld. Such information is necessary in order to directly assess the degree of the phase coupling between Fouricr components. The main arguments in support of the soliton gas hypothesis are summarized as follows:
1) At sufficiently large values of time lag, 20 days $\leq \tau$ $<80$ days, our function $W(r, \tau)$ preserves its shape as $\tau$ continues to increase, see Fig. 6. (The upper bound on $\tau$ represents the time taken by a signal to pass $8 \cdot 10^{3} \mathrm{~km}$ at speed $1.2 \mathrm{~m} / \mathrm{s}$.) We interpret this observation as an indication that wave dispersion is balanced by wave nonlinearity - as expected of a collection of solitons.

2) The observed propagation speed, $\bar{c} \approx 1.1 \mathrm{~m} / \mathrm{s}$, appcars to be higher than the prediction of linear theory.

3) A power-law spectrum $\mathrm{k}^{-2}$ of westward propagating waves with length scales up to $8 \cdot 10^{3} \mathrm{~km}$ - comparable to the basin size - is compatible with a set of compact, soliton-type formations. This spectrum was also predicted theoretically by Osborne (1983) for a set of random solitons in the presence of a radiation background, based on analysis of the KdV equation with periodic boundary and random initial conditions.

In the absence of higher-order statistical moments we cannot eliminate a possibility of weak wave turbulence as being responsible for the observed spectrum. IIowever, at the present time, estimation of bispectra or other characteristics is impeded by the relatively low measuring accuracy, limited spatial and temporal resolution and still insufficient volume of SSH observations. The use of alternative approaches, especially the nonlinear filtering technique (Osborne, 1993, 1995), might prove advantageous in future studies.

Appendix A: Linear model of Rossby and Yanai waves

Linear theory yields the following dispersion relationship for the $m$-th meridional mode Rosshy waves:

$$
\omega=-\frac{k c_{K}}{2 m+1+2(k R)^{2}},
$$

where $c_{K}$ is the Kelvin wave speed, $c_{K} \equiv \sqrt{g^{\prime} h} \approx 2.5$ $\mathrm{m} / \mathrm{s}$, and

$$
R=\left(\frac{\sqrt{g^{\prime} h}}{2 \beta}\right)^{1 / 2}
$$

is the internal Rossby radius of deformation, and $\beta \approx$ $2.3 \cdot 10^{-11}\left(\mathrm{~m} \mathrm{~s}^{-1}\right.$. In the case of a two-layered occan, $g^{\prime}$ is the reduced gravity and $h=H_{1} H_{2} /\left(H_{1}+H_{2}\right)$ where $\mathrm{H}_{1}$ is the thermocline depth and $\mathrm{H}_{1}+\mathrm{H}_{2}$ is the total ocean depth, $H$. For the first vertical mode in a continuously stratified ocean, $h$ in (A2) is replaced with $(\mathrm{NH} / / \pi)^{2} / \mathrm{g}$ where $\mathbf{N}$ is the Brunt-Väisälä frequency, and $\mathrm{g}^{\prime}$ is replaced with $\mathrm{g}$. We are concerned with the case when the Rossby radius is small - as specified later in this section. For brevity, let us re-denote the Rossby wave phase speed $c_{R, m}(k)$ by $c(k)$ and $c_{R, m}(0)$ by $c_{0}$. For long Rossby waves, the dispersion relationship can be simplified by assuming $(R k)^{2}<m+1 / 2$ for $k$ in the range $\left.\left(k_{0}\right), k_{\infty}\right)$. Thus, the phase speed is

$$
c(k)=c_{0}\left(1-\varepsilon k^{2}\right) \text {. }
$$

where 


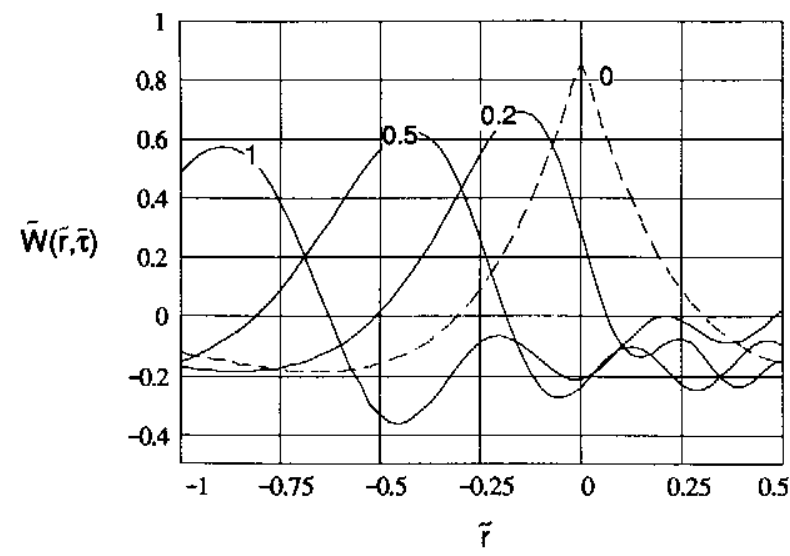

Fig. A1. The non-dimensional spatio-temporal autocorrelation function (A5) (normalized to unity at $r=0$ ) for linear Rossby waves with spectrum (A7). Horizontal axis: the non-dimensional spatial lag, $\tilde{r}$, (scaled by $L=5 \cdot 10^{3} \mathrm{~km}$ ). Other scales are indicated in Appendix A. Numbers at the curves are values of $\bar{\tau}$ with time scale $L / c_{0} \approx 70$ days, Dashed curve: $\tilde{\tau}=\mathbf{0}$.

$$
c_{0}=-\frac{\sqrt{g^{\prime} h}}{2 m+1}, \quad \varepsilon=\frac{R^{2}}{m+1 / 2}
$$

Let us write (3) with (A3) in a non-dimensional lorm

$$
\tilde{W}(\tilde{r}, \tilde{\tau})=\int_{0}^{\infty} \tilde{F}(\kappa) \exp \left[i Q \kappa(\tilde{r}-\tilde{\tau})+i \tilde{\varepsilon} \tilde{\tau} \kappa^{3}\right] d \kappa
$$

where the non-dimensional variables are related to $R, c_{0}$ and $\mathrm{k}_{0}$ by the following scaling relationships:

$$
\begin{aligned}
& \kappa=k / k_{0}, \quad \tilde{r}=r / L, \quad \tilde{\tau}=\frac{c_{0}}{L} \tau, \\
& Q=k_{0} L, \quad \tilde{\varepsilon}=\frac{\left(k_{0} R\right)^{2} Q}{m+1 / 2} .
\end{aligned}
$$

Here, the spatial length scale $L$ is arbitrary. Sclecling $L=$ $5 \cdot 10^{3} \mathrm{~km}, k_{O} \approx 2 \pi \cdot 10^{-4} \mathrm{rad} / \mathrm{km}$ and $c_{K}=2.5 \mathrm{~m} / \mathrm{s}$, wc have for $m=1: \quad R=233 \mathrm{~km}, c_{O} \approx 0.83 \mathrm{~m} / \mathrm{s}$ and $\tilde{\varepsilon} \approx 0.04$. These values of $c_{K}$ and $R$ are typical for the equatorial Pacific (LeBlond and Mysak, 1978). The corresponding time scale $L / c_{O} \approx 70$ days.

The non-dimensional version of $(6)$ is

$$
F(\kappa)=\exp \left(-1 / \kappa^{2}\right) \kappa^{-2}
$$

(We omit the low-pass filter in (6) because the integral $(\Lambda 5)$ converges sufficiently fast.) In Fig. A1, the real part of (A5) is plotted based on numerical integration. Nt timc moment $\tilde{\tau}=1$ (corresponding to 70 days), the maximum of $\mathrm{W}(r, \tau)$ is found at a point $\tilde{r} \approx-0.87$. In the absence of wave dispersion it would be at a point $\tilde{r}=-1$. This delay allows one to estimate the ratio of the longest-Rossby-wave speed to the propagation speed of the autocorrelation maximum (5):

$$
c_{0} / \bar{c} \approx 1 / 0.87 \approx 1.15 \text {. }
$$

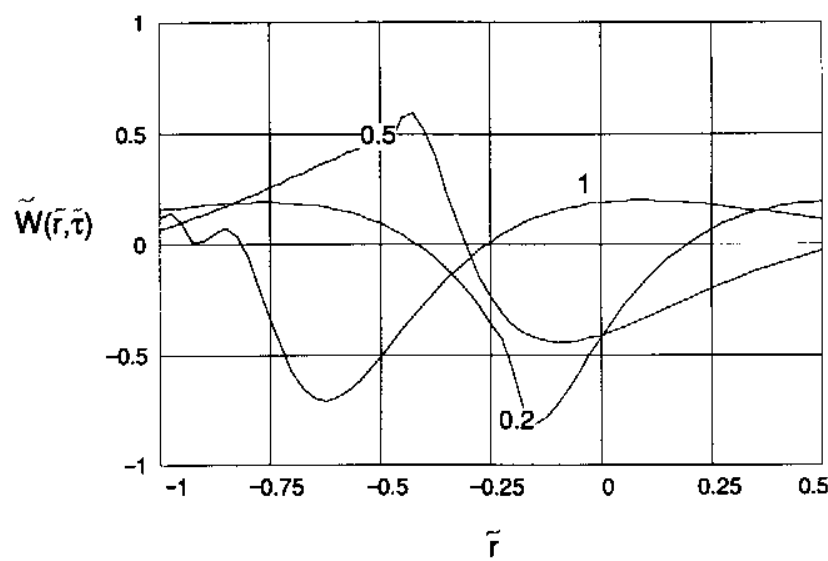

Fig. A2. The same as Fig.A1, but for linear Yanai waves with $v=0.14$. Numbers at the curves are values of $\tilde{\tau}$. The curve for $\tilde{\tau}=0$ is not plotted - as it is identical to the dashed curve in Fig. Al.

In order to show that the mixed Rossby-gravity mode, $m=0$, cannot be responsible for the observed $W(r, \tau)$, one may use the (non-dimensional) dispersion relationship for these waves, $\tilde{\boldsymbol{\omega}}=\left[1+\sqrt{1+2 /(v \tilde{k})^{2}}\right] / 2$, in the lincar model analogous to (A5). Here, $v=k_{0} R$. The result is shown in Fig. A2 (all scaling parameters as the sanc as for Fig. A1). Evidently, the broad-band spectrum of the Yanai waves is inconsistent with our estimate of the spatiotemporal autocorrelation function.

\section{Appendix B: An overview of equatorial Rossloy solitons}

We present here a brief summary of basic notions related to soliton turbulence. For a more technical accoumt of this material, the reader is referred to (Boyd 1980, 1985, 1991) and (Osborne 1983, 1985).

For odd-numbered meridional modes, non-lincar theory of equatorial Rossby waves (Boyd 1980, 1985) yiclds a single-bump soliton or a modon - provided the amplitude is above a certain threshold. In either case, the zonal variation of the surface topography (or of the zonal component of the velocity field) is given by

$$
A(x, t)=12 s^{2} \operatorname{sech}^{2}[s(x-c t)]
$$

where $s$ is a constant, and the propagation speed, $c$, exceeds $c_{R, m}$ by an amount proportional to the wave anplitude. In the case of solitons, the latitudinal variation of pressure (hence, sea level) and velocity fields is the samc as lor linear waves (Moore and Philander, 1977). lior equalorial modons the latitudinal structure is more complicaled: wave field kinematics are characterized by dipolar vorticity (Iwo equal and opposite centers of rotation), closed slreaklines, etc. - in close resemblance to mid-latitude mokons (Boyd, 1985, 1991).

Under periodic boundary conditions, the KalV equation yields cnoidal waves: 


$$
A(x, t)=-24 s / \pi+\sum_{n=-\infty}^{\infty} 12 s^{2} \operatorname{sech}^{2}[s(x-c t-n \pi)]
$$

The fact that this solution can be viewed as a sum of evenly spaced solitons (compare with (B1)) was used by Boyd (1991) to promote a concept of "soliton trains." $\Lambda$ stochastic version of this concept requires a random distribution of soliton phases and amplitudes. I.et us notice that, while the phases of individual solitons may be uniformly distributed in the interval $(-\pi, \pi)$ - hence not coupled to each other - the phases of individual Fouricr harmonics comprising each hump are strongly coupled. The physical meaning of the Fourier phases and of the soliton phases is thus quite different. Only in the limit of linear waves - when the hyperbolic functions in (B2) degenerate into cosinc functions - will the phases of individual wave crests and the phases of Fouricr harmonics have a similar physical meaning (Osbornc, 1993). Gorshkov et al. (1977), D'yachenko et al. (1989) and other authors studying stochastic solutions of nonlincirr cqualiows.s with weak dispersion used the terms "soliton gas" or "soliton turbulence." These tcrms appear to he morc relevant to our case than Boyd's "soliton train."

When wave dispersion is greater than wave nonlincarily, solitons do not appear and the phases of individual Fourier components are almost independent of each other. This: regime is called weak wave turbulence (Zakharov et al., 1992).

Osborne $(1983,1985)$ studied stochastic solutions (due to random initial conditions) for the KJV equation hased on inverse scattering theory. In particular, he slowwed that a set of (nonlinearly interacting between lhemselves and with the background of transient disturbances) solitons with uniformly distributed soliton phases is characlerized by wavenumber spectrum $\mathrm{k}^{-2}$ - in agrecmenl with our observations. Furthermore, individual solitons do not have to be widely separated in space to produce the $k^{-2}$ spectrun. The hyperbolic functions in (B2) are replaced wilh hyperelliptic functions. The fact that the latter reduce to cosine functions in the limit of vanishing nonlinearity led Osborne to view the soliton gas as a nonlincar generalization of a Fourier series.

For even-numbered meridional modes, the appropriate equation for zonal motion is a "Modified KdV" (Boyd, 1980) whose nonlinear term is $\Lambda^{2} \Lambda_{x}$ raller (han $\Lambda \Lambda_{x}$. This equation describes a wave motion whose degree of nonlinearity is lower than that given by the standard $\mathrm{KdV}$ equation. As a result, MKdV yields neither solitons nor modons. The latter may exist only for odd-numbered latitudinal modes.

Acknowledgments: This work was performed at the Jet Propulsion Laboratory, California Institute of Technology, under contract with the Nationul Aeronatulics and Space Administration. The authors thank Prof'. L. Pilerthurg of the Math. Dept. at USC and Prof. A. Balk of the $\Lambda_{\text {ppl. Muth. Dept. }}$ at Caltech for valuable comments.

\section{References}

Abramowitz, M., and I.A. Stegun, Handbook of Maihematical Functions, 1045 pp. Nat. Inst. of Standards and Technology, U.S. Department of Commerce. (iaithershurg, MD., 1970.

Balk, A.M. and S.V. Nazarenko, Physical realizulility of anisotropic weak-turbulence Kolmogorov spectru. Sov. Phys. JETP, 70(6), 1031-1040. 1990.

Benada, R., Internal Publication of the Jet l'ropulsion Laboratory, D-11007 "Merged GDR (Topex/l'oseidon) Users Handbook, Version 1.", September, 1993.

Boyd, J.P. Equatorial solitary waves. Part I: Rosshy sulituns. Journ. Phys. Oceanogr., 10(11), 1699-1717, 1980.

Boyd, J.P. Equatorial solitary waves. Part 3: Westwardtraveling modons. Journ. Phys. Oceanogr., 15(1), 46-54, 1985.

Boyd, J.P. Nonlinear equatorial waves. In 'Nonlinear Topics of Ocean Physics" , pp. 51-97. Edited by A.R. Oslxo'ne and i.. Bergamasco. North-Holland, Amsterdam, 1991.

Delcroix T., Picaut J., and Eldin G. licyuatorial Kelvin and Rossby waves evidenced in the Pacific ocean thowgh (ieosal sea level and surface current anomalies. J. Gi('ophys. Re's., Oceans. 96, 3249-3262. 1991.

D'yachenko, A.I., V.E. Zakharov, A.N. Pushkurev, V.l;. Shvets, and V.V. Yan'kov. Soliton turhulence in $1101-$ integrable wave systems. Sov. Phys, JLTP $69(6)$, I/441147, 1989.

Fu, L.L, E.J. Christensen, C.A. Yamarone Jr., M. I.etelevre, Y. Menard, M. Dorrer, and P. Escudier. TOPliX/POSIIIION mission overview. J. Geophys. Res., 99(C12), 24,369. 24,381, 1994.

Glazman, R.E., and P. Weichman. Statistical geometry of a small surface patch in a developed sea. J. Geophys. Res., 94(C4), 4998-5010, 1989.

Glazman, R.E. , A. Fabrikant, and A. Greysukh Stutistics of spatial-temporal variations of sea surface height haxed on Topex altimeter measurements. Int. J. Remote Senxing. (In press). 1996.

Gorshkov, K.A., L.A. Ostrovskii, and V.V. Papko. Soliton turbulence in a system with weak dispersion. Sov. Phys. Dokl. 22(7), 378-380, 1977.

Kingsep, A.S., L.I. Rudakov, and R.N. Sudan. Phys. Rev. lett. $31,1482,1973$.

LeBlond, P.H. and L.A. Mysak. Waves In The Ocean. FIsivier, New York, 1978, 602 pp.

Marshall, H.G. and J.P. Boyd. Solitoms in a continuously stratified equatorial ocean. Journ. Phys. Oreanogr., /7(7), 1016-1031, 1987.

Mikhailovskii A.B., Novakovskii S.V., I.ukliin V.P., Makurin S.V., Novakovskaya Ji.A.. Onishchenko O.G. Kolmogorov weak turbulence spectra for inbomogeneous magnetized plasma. Sov. Phys. JETP. 67, 1.386-1.187. 1988.

Mikhailovskii A.B., Nazarenko S.V., Novakovkkii S.V., Churicov A.P., Onishchenko O.G., Kolmogorov weakly turbulent spectra of some types of drift waves in plasmas. Phys. Lett. A, 133(7-8), 407-409, 1988.

Monin, A.S. and L.I. Piterbarg. A kinetic equation for Rosshy waves. Sov. Phys. Dokl., 32(8), 622.624, 1987.

Moore, D.W. and S.G.H. Philander. Chapter 8: Modleling of the Tropical Pacific Circulation. In "The Sea", 6, edited hy I..D. Goldberg. Wiley, N.Y., 1977.

Musman, S. Geosat altimeter ohservations of long waves in the equatorial Atlantic. J. Geophys. Res., 97(C:3), 3.5.37$3579,1992$. 
Novakovskii S.V., Mikhailovskii A.B., Onishchenko O.G. On the theory of Kolmogorov spectra of drift wave turbulence. Phys. Lett. A 132(1), 33-38, 1988.

Osborne, A.R. Behavior of solitons in random-function solutions of the periodic Korteweg-de Vries equation. Phys. Rev. Lett., 71(19), 3115-3118, 1993.

Osborne, A.R. Solitons in the periodic Korleweg-de Vries equation, the $\Theta$-function reprexentution, and the annlysix of nonlinear, stochastic wave trains. Phys. Rev. L: 52(I), 1105-1122, 1995.

Tai, C.-K. Accuracy assessment of wiclely used orhit error approximations in satellite altimetry ankl its oceunographic implications, J.Atm Oceanic Techn., 6, 147.150. 1989.

Zakharov, V.E., V.S. I.'vov and (i. l'alkuvich. (')!)2. Kolmogorov Spectra of Turbulence I: Wave Turbulence. Springer Verlag, Berlin, $264 \mathrm{pp}$. 